\title{
My MFFP dissertation
}

\author{
Amanda Smith, MBBS, MRCGP, DRCOG, DFFP, CMO and Career Grade Trainee
}

Correspondence: Amanda Smith, Contraception and Sexual Health Services, Community Health Care Services (North Derbyshire) NHS Trust, Saltergate Health Centre, Chesterfield, North Derbyshire, S40 15X, UK

(Accepted July $\left.26^{\text {th }}, 2000\right)$

\begin{abstract}
Summary
This paper describes how Amanda Smith set about her MFFP dissertation, from start to finish, and asks the question 'Was it all worth it?'
\end{abstract}

\section{Key words}

MFFP dissertation, research methods

\section{Choosing my subject}

The most difficult part was getting started. I did not have a burning clinical question that was answerable in the time scale I had set myself. I wanted my chosen project to be in an important area of my day to day work, and to lead to improvements in the service to our clients. I hoped that my dissertation would develop my skills in new (non-clinical) directions and help me to complete as many areas of the logbook as possible in the Career Grade modular training programme, should I be appointed to such a training post in the future. You are probably wondering (as I was at the time) how I could achieve all these objectives.

I toyed with a few subject ideas. The ensuing literature searches were time-consuming. However this time was not wasted. I have subsequently referred to many of the references that I gathered even for the ideas that I did not pursue. I decided to do a contraception and sexual health needs assessment in a small town where I represent the medical input to a weekly community family planning clinic.

\section{Getting help}

Many people were happy to give advice. My consultant knew several informative colleagues. The Trust's Clinical Audit and Research 'Team' gave me helpful advice about methodology, how to make an application to the Ethics Committee, and how to get free advice sessions from research advisers and statisticians at a nearby research centre. They also put me in touch with other members of staff who had done previous needs assessment work.

I filled in and sent the 'Intention to Submit a Dissertation' application form to the Faculty, with the appropriate fee. This was a relatively simple procedure. I was duly appointed a supervisor who came to see me at home and gave me more useful advice on the standard required and on the pros and cons of various research methods.

\section{Choosing my methods}

I soon realised that there were many more issues to consider. My original project was overly ambitious. I had to scale down its size and consider just the needs of one group, namely young people under 25 . The methods to be used would need to be appropriate to the questions that I wished to answer. They would also have to be practical in terms of the time that I had available to do the project. At that time my salaried post did not include any paid sessions for activities such as research. I would have to fit meetings, interviews and discussion groups around my regular work sessions, which would eat into the time that I usually spent with my three-year-old son.

Would I need funding? If I used research workers to help with the project this would increase the costs. Applying for funds could be a lengthy process and success would not be guaranteed with the first application. Choosing methods that I could undertake myself would be financially cheaper, but expensive on my own personal time. A subject needing Ethical Committee approval would allow me to learn about the process, but would increase the time and effort involved in doing the study.

My chosen project involved liaison with other professionals, including youth workers and teachers. This approach would help develop my communication and project management skills and help to increase local awareness of the project. There were to be drawbacks as well. The lead youth worker at the youth club where my project was based moved to a new job a hundred miles away just before my questionnaires were sent out to young people in youth clubs and schools. The vacant post was not filled for over a year. A youth worker in the neighbouring village very kindly gave me much help. However, the lack of a lead youth worker where the project was based left a gap in local knowledge and to considerable delays. Short school terms and long summer holidays were an additional barrier to obtaining enough questionnaire responses.

\section{Trying to make progress}

I became pregnant, expecting my second child. I thought I had plenty of time to get enough questionnaire responses, and enough young people recruited to the focus groups I had planned. I even thought that I would have enough time to write up my dissertation before the birth. However, I had not allowed for the difficulties that I would encounter in trying to get young people to come to the focus groups. It was a triumphant day when finally the groups took place. I had great support from Sue, a local youth worker, from Lindsay, one of our talented nurses and from Darren and Richard, two local project workers.

I found it difficult to motivate myself to finish analysing the mass of qualitative information I had accumulated. Looking back, I think this was due to the extreme tiredness of being 8 months pregnant! It was also Christmas...

In early January I went back to the youth club to see focus group members to validate some data. I heard one young man whisper to another 'is she going to have a baby?' I stated that I was pregnant but that I wasn't planning to have the baby that day (my EDD was still 3 weeks away). This information seemed to relieve his anxiety. Little did he 
know that a few hours later my 'waters' would gush all over the utility room floor when I knelt down to load the washing machine!

After my second son was born, I actually felt more motivated to finish my project. Indeed, I became very skilled at typing with one hand whilst breastfeeding. We whiled away the early hours of several mornings in this way...

\section{Acting on the results}

When I returned to work, the clinic staff met to discuss the project's findings and agree roles and time scales to implement changes. We invited local professionals to a meeting to inform them of our service and the improvements we had made. This was combined with a campaign of posters and leaflets in places suggested by the young people who participated.

\section{Evaluating it all}

There has been an increase in numbers attending the new service. A satisfaction survey is planned for autumn 2000.
I am told that problems such as I encountered are common, and that the path of research is not smooth. I could have done something simpler. I'm sure that a lot of hard work and frustration would still have been required. I did achieve all my objectives. I have learnt far more about needs assessment, qualitative research methods, project management, liaison with health promotion, schools and youth services, teenage pregnancy, sex education and youth culture from this project than I could have done from a book or a training course. Was it all worth it (as the rock group Queen asked of 'living, breathing rock and roll' in their album 'The Miracle')? Yes it was worth it; it was a worthwhile experience!

\section{Acknowledgments}

Thanks to Hilary Richards and Sarah Hughes for their helpful feedback on the initial draft of this piece.

\section{From the examination committee}

The following flow chart is sent to all candidates to assist them in the preparation of their dissertation:

\section{OUTLINE OF PROCEDURE}

'Intention to Submit a Dissertation' form and 'Guidance Notes for Candidates' from the Faculty

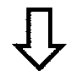

Candidate returns completed form (which includes a summary of the proposed project) to the Faculty, to be assessed and approved by the Dissertation Convenor
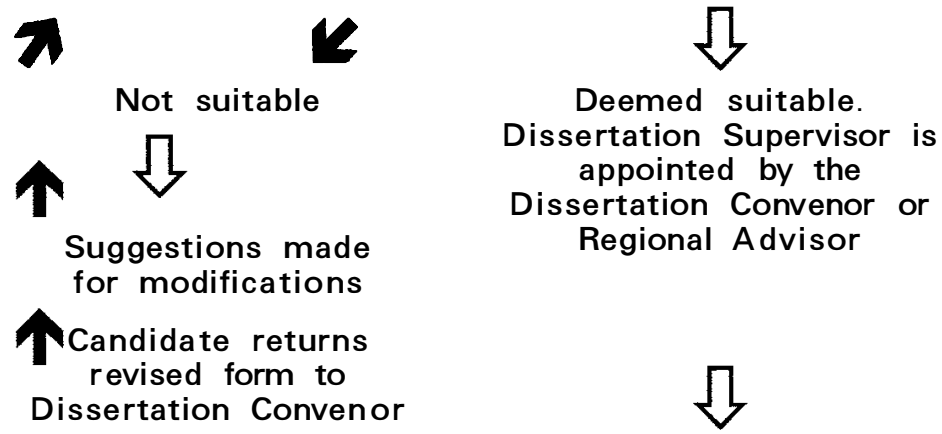

Regional Advisor 